\title{
THE EFFICIENCY OF A BONUS-MALUS SYSTEM
}

\author{
Nelson IEe PriL \\ Belgium
}

\begin{abstract}
The concept efficiency of a bonus-malus system was defined, apparently in a totally different way, consecutively by Loimaranta (I972) and Lemaire (1975, 1976). In this paper we start with a more general model that leads us to a clefinition of efficiency that contains both earlier ones as special cases. Further we introduce the definition of efficiency over a finite planning horizon and consider the efficiency not only for a single risk but also for the entire risk group. As a consequence of our approach we can also generalize the concepts excess premium and central value as they were introduced by Loimaranta.
\end{abstract}

\section{The Bonus-Malus System as Markov Chain}

The basis of a fair tarification in insurance, in our case motorcar insurance, consists in the fact that each policyholder is charged a premium that is proportional to the risk that he actually represents. This risk is determined by a great number of risk factors. Some of them, such as type and use of the car, can be taken into consideration a priori for the tarification and they enable us to split up the heterogeneous collectivity of risks into a number of risk groups which have a more homogeneous risk structure. Other factors cannot be taken into account a priori since they are too difficult to observe, or for social and psychological reasons, or just because one doesn't know all the factors which influence the risk. Due to these factors there will still be accident proneness differentials within a risk group. In the course of time these differentials will be reflected by the individual claim experience of the risk. Therefore one can bring into account a posteriori the earlier neglected risk factors by means of an individual experience rating method, such as a bonus-malus system.

From a point of time $t=0$ we consider such a risk group in which the tarification is based on a bonus-malus system that is determined by the following factors.

- The length of an insurance period is $I$, which means nothing else than that the length of a period is choosen as unit of time.

- The number of classes is $n$.

- The premium which a risk of class $j$ has to pay at the moment $t$ 
to be insured for the period $\left[t, t+\mathrm{I}\left[\right.\right.$ is $b_{t}(j) ; j \varepsilon\{\mathrm{I}, \ldots, n\}, t \varepsilon$ $\{0, I, \ldots\}$.

- The initial class in which a risk is placed at $t=0$ is the class $s$.

- The transition rules are given in the form of probabilities $t_{i j}(k)$; $i, j \varepsilon\{\mathrm{I}, \ldots, n\}, k \varepsilon\{0, \mathbf{I}, \ldots\}$; where $t_{i j}(k)=\mathrm{I}$ if a risk of class $i$ moves to class $j$ when $k$ claims have occurred in the past period, and $t_{i j}(k)=0$ if such a risk goes to a class different from $j$. In order that the transition rules be complete and free of contradictions we must have: for each $(i, k)$ there is one and just one $j$ so that $t_{i j}(k)=\mathrm{x}$.

We assume that the accident proneness of a risk of the considered risk group can be represented by a risk parameter $\lambda$, which is the claim frequency of the risk, i.e. the expected number of claims per period for that risk. The value of the risk parameter is regarded as a realization of a random variable $\Lambda$, whose distribution function $U(\lambda)$ represents the risk structure of the group. We take that the value of the risk parameter is independent of time. Further we assume that for a given risk $\lambda$ the random variables which give the number of claims for the successive periods are mutually independent and identically distributed with common probability distribution $p_{k}(\lambda)$, which depends explicitely and uniquely on the parameter $\lambda$.

These assumptions permit us to describe the evolution of a given risk through the bonus-malus system by a Markov chain with constant transition matrix. The probability $p_{i j}^{(t)}(\lambda)$ that a risk $\lambda$ which is in the class $i$ will be in the class $j t$ periods later, is given by the recursion formula

$$
\left\{\begin{array}{l}
p_{i j}(\lambda)=\sum_{k=0}^{\infty} p_{k}(\lambda) t_{i j}(k) \\
p_{i j}^{(t)}(\lambda)=\sum_{r=1}^{n} p_{i r}(\lambda) p_{r j}^{(t-1)}(\lambda) \quad t=2,3, \ldots
\end{array}\right.
$$

\section{The Efficiency of a Bonus-Malus System}

One notices that each country and in some countries even each insurance company has its own bonus-malus system. However all this systems have the same purpose, viz. to come to a fair tarification by adjusting the premiums of each individual policyholder as good as possible to the risk that he actually represents. To measure how good a system fulfils this requirement the concept efficiency is introduced. 
We denote by $X_{\tau}(\lambda)$ a random variable that gives the discounted value of all premiums that will be paid by a risk $\lambda$ in the time interval $[0, \tau[, \tau\{t, 2, \ldots\}$.

These premiums are the ones paid at the moments $O, I, \ldots, \tau-\mathrm{I}$; where the premium at a moment $t$ equals $b_{t}(j)$ if the risk is in class $j$ at the moment $t$, and is zero if the risk has by that time left the system. The expectation $E\left[X_{\tau}(\lambda)\right]$ of the discounted value of these premium payments, which is determined by the used bonus-malus system, can be called the bonus-malus premium for a risk $\lambda$ in $\left[0, \tau\left[\right.\right.$. By $Y_{\tau}(\lambda)$ we denote a random variable that gives the discounted value of all claim costs of a risk $\lambda$ in [o, $\tau[$. The expectation $E\left[Y_{-}(\lambda)\right]$ of the discounted value of these claim costs represents the risk preminm for a risk $\lambda$ in $[0, \tau[$.

To verify how good the premium of a certain policy holder corresponds with the risk that he represents we measure the sensibility of the bonus-malus premium by changing risk premium. Therefore we compare a relative variation $\frac{d E\left[Y_{\tau}(\lambda)\right]}{E\left[Y_{\tau}(\lambda)\right]}$ in the risk premium with the relative variation $\frac{d E\left[X_{\tau}(\lambda)\right]}{E\left[X_{\tau}(\lambda)\right]}$ in the bonus-malus premium that it implies. By definition we call efficiency of a bonusmalus system for a risk $\lambda$ in $[0, \tau[$ the ratio of these two quantities

$$
e_{\tau}(\lambda)=\frac{\frac{d E\left[X_{\tau}(\lambda)\right]}{E\left[X_{\tau}(\lambda)\right]}}{\frac{d E\left[Y_{\tau}(\lambda)\right]}{E\left[Y_{\tau}(\lambda)\right]}}=\frac{d \ln E\left[X_{\tau}(\lambda)\right]}{d \ln E\left[Y_{\tau}(\lambda)\right]}
$$

The efficiency in $[0, \tau[$ is thus the elasticity of the bonus-malus premium in $[0, \tau[$ with respect to the risk premium in $[0, \tau[$. Put into words this means that for a risk $\lambda$ a variation of $\mathrm{I} \%$ in the expectation of the discounted claim costs in $[0, \tau[$ causes a variation of $e_{\tau}(\lambda) \%$ in the expectation of the discounted premium payments in $[\mathrm{O}, \tau[$.

When we take in $(2)$ the limit for $\tau \rightarrow \infty$ we get the efficiency in $[0, \infty[$, viz.

$$
e(\lambda)=\lim _{\tau \rightarrow \infty} e_{\tau}(\lambda)
$$

A first analysis of the definition of efficiency enables us to make the following observations

A reasonable bonus-malus system got to have a separation effect, so that in an average sense good risks pay lower premiums than 
bad ones. This means that the relative variations in bonus-malus and risk premium got to have the same sign, so that for each $(\lambda, \tau)$ holds $e_{\tau}(\lambda) \geq 0$.

The limitcase of a bonus-malus system in which the bonus-malus premium in $[\mathrm{o}, \tau[$ remains the same for each risk, corresponds with $e_{\tau}(\lambda)=0$ for each $\lambda$. This case shows up during the first period when each risk is in the initial class, so that we have $e_{1}(\lambda)=$ o for each $\lambda$.

The ideal case in which for each risk and for each interval the bonus-malus premium equals the risk premium corresponds with $e_{\tau}(\lambda)=\mathrm{I}$ for each $(\lambda, \tau)$. In particular $e(\lambda)=\mathrm{I}$ corresponds with an asymptotical correct tarification for a risk $\lambda$. The conditions of an ideal system can in general never be met.

In practice a relative increase in the risk premium will generally cause a smaller relative increase in the bonus-malus premium, which means that the good risks have to pay for the bad ones. In general $e_{\tau}(\lambda)$ will thus lie between the values zero and one. Theoretically we can have $e_{\tau}(\lambda)>I$ but such a case of overefficiency in which an increase in the expectation of the claim costs is more than compensated by the increase of the expectation of the premium payments is rarely found.

Because of:

$$
\begin{aligned}
& E\left[X_{\tau}(\lambda)\right]>0 \quad, E\left[Y_{\tau}(\lambda)\right] \rightarrow 0 \text { for } \lambda \rightarrow 0 \\
& E\left[X_{\tau}(\lambda)\right] \text { bounded, } E\left[Y_{\tau}(\lambda)\right] \rightarrow \infty \text { for } \lambda \rightarrow \infty
\end{aligned}
$$

We have in general that for each $\tau$ :

$$
\lim _{\lambda \rightarrow 0} e_{\tau}(\lambda)=0 \text { and } \lim _{\lambda \rightarrow \infty} e_{\tau}(\lambda)=0
$$

Geometrically the definition-formula (2) can be interpreted in the following way

$$
e_{\tau}(\lambda)=\frac{E\left[Y_{\tau}(\lambda)\right]}{E\left[X_{\tau}(\lambda)\right]} \frac{d E\left[X_{\tau}(\lambda)\right]}{d E\left[Y_{\tau}(\lambda)\right]}=\frac{\operatorname{tg} \alpha}{\operatorname{tg} \beta}
$$

So far the efficiency was defined for a risk with given and known risk parameter $\lambda$. The assumption that the risk parameter is known is useful for the development of the theory but is never fulfilled in practice. On the other hand the distribution function of the risk parameter, viz. the structure function $U(\lambda)$, is more likely known, so that it is natural to define the efficiency over the considered risk group. We call efficiency of a bonus-malus system over a given risk group in $[0, \tau[$ the expression:

$$
e_{\tau}=\int_{\Lambda} e_{\tau}(\lambda) d U(\lambda)
$$




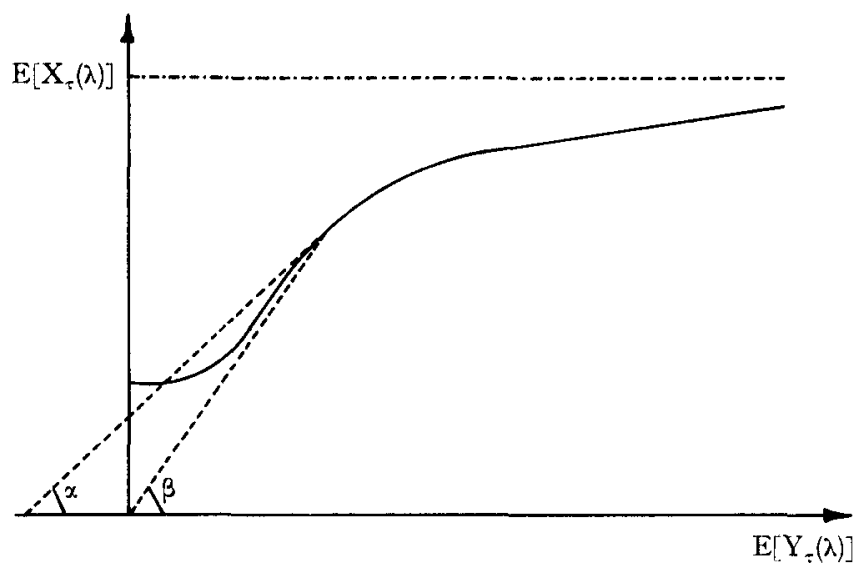

and we get for $\tau \rightarrow \infty$ :

$$
e=\int_{\Lambda} e(\lambda) d U(\lambda)
$$

This averaged efficiency over the risk group enables us to compare the different bonus-malus systems in an objective way.

Further we notice that in our definition of efficiency we could also take into account the so called "bonus-hunger effect" (cfr. Lemaire). This can be done by changing the definition of $X_{\tau}(\lambda)$ and $Y_{\tau}(\lambda)$ in an appropriate way.

Finally we remark that our concept of efficiency is not only valid for bonus-malus systems but can be applied to other experience rating systems.

\section{Calculation of the Efficiency under Different Assump- tions Concerning the Risk Process.}

3.I. We consider a risk $\lambda$ which is placed in class $s$ at $t=0$ and assume that at the end of each period this risk can either take an insurance for the next period or leave the system. By $w_{t}(\lambda)$ we denote the probability that the risk $\lambda$ is insured for the period $[t, t+\mathrm{I}[$. We take that a risk $\lambda$ which left the system cannot reenter it, so that $w_{0}(\lambda)=\mathrm{I} \geq w_{1}(\lambda) \geq w_{2}(\lambda) \geq \ldots$. Further we suppose that the average cost of a claim is independent of the number of claims and we denote by $C_{t}(\lambda)$ the average cost of a claim for a risk $\lambda$ in the period $[t, t+\mathrm{I}$ [. Finally we denote by $\beta \leq \mathrm{I}$ a discount factor. 
Under these assumptions we get for the bonus-malus premium of a risk $\lambda$ in $[0, \tau[$ :

$$
E\left[X_{-}(\lambda)\right]=\sum_{t=0}^{--1} \beta^{t_{w}}{ }_{t}(\lambda) \sum_{j=1}^{n} p_{s j}^{(t)}(\lambda) b_{t}(j) \text { with } p_{s j}^{(0)}=\delta_{s j}
$$

and we have for the risk premium

$$
E\left[Y_{\tau}(\lambda)\right]=\lambda \sum_{t=0}^{\tau-1} \beta^{t+1 / 2} \omega_{t}(\lambda) C_{t}(\lambda)
$$

Using these formulae the efficiency can in principle be calculated. However, additional assumptions concerning the earlier mentioned elements of the risk process seem desirable in order to come to a more manageable expression.

3.2. We suppose now that for each period $[t, t+\mathrm{I}[$, both the probabilities $w_{t}(\lambda)$ and the average claim costs $C_{t}(\lambda)$ are the same for all risks of the considered risk group, this is that they are independent of the parameter $\lambda$.

Under these assumptions formula (2) is reduced to

$$
e_{\tau}(\lambda)=\frac{d E\left[X_{\tau}(\lambda)\right]}{E\left[X_{\tau}(\lambda)\right]} \frac{\lambda}{d \lambda}
$$

More explicitly we have

$$
e_{\tau}(\lambda)=\frac{\sum_{t=0}^{\tau-1} \beta^{t} w_{t} \sum_{j=1}^{n} \frac{d p_{s j}^{(t)}(\lambda)}{d \lambda} b_{t}(j)}{\sum_{t=0}^{-1} \beta^{t} w_{t} \sum_{j=1}^{n} \frac{p_{s j}^{(t)}(\lambda)}{\lambda} b_{t}(j)}
$$

where the derivatives are determined by the recursion formula

$$
\left\{\begin{array}{l}
\frac{d p_{i j}(\lambda)}{d \lambda}=\sum_{k=0}^{\infty} \frac{d p_{k}(\lambda)}{d \lambda} t_{i j}(k) \\
\frac{d p_{i j}^{(t)}(\lambda)}{d \lambda}=\sum_{r=1}^{n}\left[\frac{d p_{i r}(\lambda)}{d \lambda} p_{r j}^{(t-1)}(\lambda)+p_{i r}(\lambda) \frac{d p_{r j}^{(t-1)}(\lambda)}{d \lambda}\right]
\end{array}\right.
$$

We remark that in the case that the number of claims is Poisson distributed formula (I3.a) becomes

$$
\frac{d p_{i j}(\lambda)}{d \lambda}=\sum_{k=0}^{\infty} e^{-\lambda} \frac{\lambda^{k}}{k !}\left[t_{i j}(k+\mathrm{I})-t_{i j}(k)\right]
$$


3.3. Moreover we make an assumption concerning the evolution in time of the premiums $b_{t}(j)$ and the probabilities $w_{t}$. We assume that the premium of each class will increase with the same percentage for each period, this is $b_{t}(j)=\alpha^{t} b_{j}$ with $b_{j}=b_{0}(j)$ the value of the premium of class $j$ at constant price and $\alpha \geq I$ the price index of premiums. Further we assume that the probability to leave the system at the end of a certain period is independent of the considered period and equals $\rho$, this means $w_{t}=(\mathrm{I}-\rho)^{t}$ where $\rho \varepsilon[0, I]$ is the rate of exit. We shall put $\theta=\beta \alpha(I-\rho)$, in which we take that $\theta \leq \mathrm{I}$ what is satisfied in practical cases.

Finally we suppose that the Markov chain which is associated to the bonus-malus system is regular. Then the limit probabilities

$$
a_{j}(\lambda)=\lim _{t \rightarrow \infty} p_{s j}^{(t)}(\lambda)
$$

exist and are independent of the initial class. They are uniquely defined by the system of equations

$$
\left\{\begin{array}{l}
a_{j}(\lambda)=\sum_{i=1}^{n} a_{i}(\lambda) p_{i j}(\lambda) \\
\sum_{i=1}^{n} a_{j}(\lambda)=\mathrm{I}
\end{array}\right.
$$

Under these assumptions equation (9) is reduced to

$$
E\left[X_{\tau}(\lambda)\right]=\sum_{i=0}^{\tau-1} \theta^{t} \sum_{j=1}^{n} p_{s j}^{(t)}(\lambda) b_{j}
$$

and if we put

$$
\begin{gathered}
b(\lambda)=\sum_{j=1}^{n} a_{j}(\lambda) b_{j} \\
g_{s,--1}(\lambda)=\sum_{i=0}^{\tau-1} \theta^{t} \sum_{j=1}^{n}\left[p_{s j}^{(t)}(\lambda)-a_{j}(\lambda)\right] b_{j}
\end{gathered}
$$

we get

$$
E\left[X_{\tau}(\lambda)\right]= \begin{cases}\frac{\mathrm{I}-\theta^{\tau}}{\mathrm{I}-\theta} b(\lambda)+g_{s, \tau-1}(\lambda) & \theta<\mathrm{I} \\ \tau b(\lambda)+g_{s, \tau-1}(\lambda) & \theta=\mathrm{I}\end{cases}
$$

We remark that

$$
\lim _{\tau \rightarrow \infty} \frac{E\left[X_{\tau}(\lambda)\right]}{\tau}= \begin{cases}0 & \theta<\mathrm{I} \\ \sum_{j=1}^{n} a_{j}(\lambda) b_{j} & \theta=\mathrm{I}\end{cases}
$$


so that $b(\lambda)$ represents for a risk $\lambda$ the limit value of the premium per period in the case $\theta=$ I. (e.g. $\left.\alpha=\frac{I}{\beta}, p=0\right)$.

The first term in (20) is the discounted expectation of the premium payments in $[0, \tau[$ for a risk $\lambda$ in the case that the premium to be insured for the period $\left[t, t+I\right.$ [ equals $b(\lambda) \alpha^{t}$, irrespective of the class in which the risk is placed at the moment $t$. The second term $g_{s, \tau-1}(\lambda)$ represents the discounted expectation of the extra premium (positive or negative) that has to be paid in $[0, \tau[$, since the premium that a risk $\lambda$ has to pay to be insured for the period $\left[t, t+\mathrm{I}\left[\right.\right.$ isn't $b(\lambda) \alpha^{t}$ but $b_{j} \alpha^{t}$, with $j$ the class in which the risk is placed at the moment $t$. This correction term depends on the initial class $s$ and $g_{s, \tau-1}(\lambda)$ is called the excess premium of the class $s$ for a risk $\lambda$ in $[0, \tau[$. The advantage of the introduction of the excess premiums lies in the fact that they simplify to a great extent the calculation of $E\left[X_{\tau}(\lambda)\right]$ and thus of $e_{\tau}(\lambda)$. It is easy to verify that for the excess premiums the following recursion formula is valid

$$
\left\{\begin{array}{l}
g_{i, 0}(\lambda)=b_{i}-b(\lambda) \\
g_{i, \tau}(\lambda)=b_{i}-b(\lambda)+\theta \sum_{j=1}^{n} p_{i j}(\lambda) g_{j, \tau-1}(\lambda) \tau=\mathrm{I}, 2, \ldots
\end{array}\right.
$$

so that it is no longer necessary to calculate $g_{s, \tau-1}(\lambda)$ from (I9), which would require the preliminary computation of all appearing $p_{s j}^{(t)}$.

Further we have that for each $(\lambda, \tau)$ the following relation hold

$$
\sum_{i=1}^{n} a_{i}(\lambda) g_{i, \tau}(\lambda)=0 \quad \tau=0, \mathrm{I}, \ldots
$$

According to (II) and (20) we get then for the efficiency in $[0, \tau[$

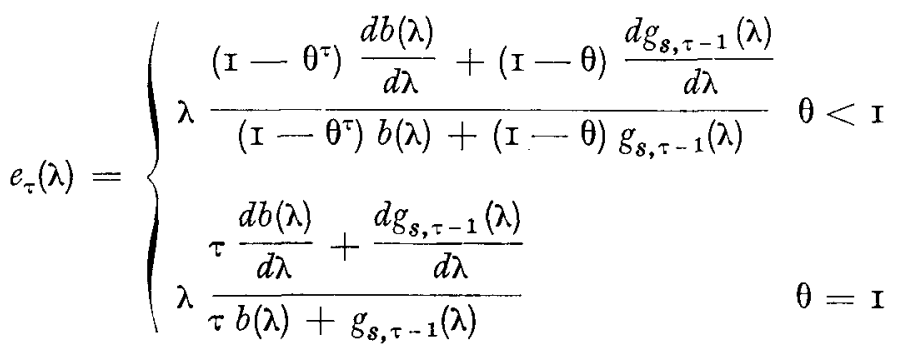

where $b(\lambda)$ can be calculated from (I6) and (I8), while $g_{\boldsymbol{s}, \tau-1}(\lambda)$ is given by (22) in which (23) is useful for control purposes. 
The derivative $\frac{d b(\lambda)}{d \lambda}=\sum_{j=1}^{n} \frac{d a_{j}(\lambda)}{d \lambda} b_{j}$ can be computed from the system of equations obtained by derivating (I6)

$$
\begin{aligned}
& \left\{\frac{d a_{j}(\lambda)}{d \lambda}=\sum_{i=1}^{n}\left[\frac{d a_{i}(\lambda)}{d \lambda} p_{i j}(\lambda)+a_{i}(\lambda) \frac{d p_{i j}(\lambda)}{d \lambda}\right]\right. \\
& \sum_{i=1}^{n} \frac{d a_{j}(\lambda)}{d \lambda}=0
\end{aligned}
$$

whereby the derivative of $p_{i j}(\lambda)$ is given by (I3.a) or (I4).

Finally the derivative of $g_{s, \tau-1}(\lambda)$ is determined by the recursion formula

$$
\left\{\begin{aligned}
& \frac{d g_{i, 0}(\lambda)}{d \lambda}=-\frac{d b(\lambda)}{d \lambda}\left\{\frac{d g_{i, \tau}(\lambda)}{d \lambda}=-\frac{d b(\lambda)}{d \lambda}+\theta \sum_{j=1}^{n}\left[\frac{d p_{i j}(\lambda)}{d \lambda} g_{j, \tau-1}(\lambda)+p_{i j}(\lambda) \frac{d g_{j, \tau-1}(\lambda)}{d \lambda}\right]\right. \\
&\tau=\mathrm{I}, 2, \ldots . \mathrm{a})
\end{aligned}\right.
$$

and the controlling equations (23) become

$$
\sum_{i=1}^{n}\left[\frac{d a_{i}(\lambda)}{d \lambda} g_{i, \tau}(\lambda)+a_{i}(\lambda) \frac{d g_{i, \tau}(\lambda)}{d \lambda}\right]=0 \quad \tau=0, \mathrm{I}, \ldots
$$

To calculate the efficiency in $[0, \infty[$, we first extend the concept excess premium to $[0, \infty[$

$$
g_{s}(\lambda)=\lim _{\tau \rightarrow \infty} g_{s, \tau}(\lambda)=\sum_{t=0}^{\infty} \theta^{t} \sum_{j=1}^{n}\left[p_{s j}^{(t)}(\lambda)-a_{j}(\lambda)\right] b_{j}
$$

Since the $p_{s j}^{(t)}(\lambda)$ converge geometrically fast to the limit probabilities $a_{j}(\lambda)$ the series $(28)$ is absolute convergent. When we take in (22.b) and (23) the limit for $\tau \rightarrow \infty$ we get the following system of equations for the excess premiums in $[0, \infty[$ of the different classes

$$
\left\{\begin{array}{l}
g_{i}(\lambda)=b_{i}-b(\lambda)+\theta \sum_{j=1}^{n} p_{i j}(\lambda) g_{j}(\lambda) \\
\sum_{i=1}^{n} a_{i}(\lambda) g_{i}(\lambda)=0
\end{array}\right.
$$

We remark that, in this system of $n+$ I equations in $n$ unknown, for $\theta<I$ the equation (29.b) is a consequence of the relations (29.a), 
while for $\theta=I(29 . b)$ is independent of (29.a) but in this case we have that the relations (29.a) are linear dependent.

In an appendix we shall prove that the $g_{i}(\lambda)$ are determined in an unique way by the system (29).

For the efficiency in $[0, \infty$ [ we have now

$$
e(\lambda)= \begin{cases}\frac{d b(\lambda)}{d \lambda}+(\mathrm{I}-\theta) \frac{d g_{s}(\lambda)}{d \lambda} & \\ \lambda \frac{b(\lambda)+(\mathrm{I}-\theta) g_{s}(\lambda)}{b} & \theta<\mathrm{I} \\ \frac{\lambda}{b(\lambda)} \frac{d b(\lambda)}{d \lambda} & \theta=\mathrm{I}\end{cases}
$$

in which $g_{s}(\lambda)$ can be calculated from (29), while its derivative is determined in an unique way (cfr. appendix) by the system of equations

$$
\left\{\begin{array}{l}
\frac{d g_{i}(\lambda)}{d \lambda}=-\frac{d b(\lambda)}{d \lambda}+\theta \sum_{j=1}^{n}\left[\frac{d p_{i j}(\lambda)}{d \lambda} g_{j}(\lambda)+p_{i j}(\lambda) \frac{d g_{j}(\lambda)}{d \lambda}\right] \\
\sum_{i=1}^{n}\left[\frac{d a_{i}(\lambda)}{d \lambda} g_{i}(\lambda)+a_{i}(\lambda) \frac{d g_{i}(\lambda)}{d \lambda}\right]=0
\end{array}\right.
$$

So we find as a special case (3o.b) the definition of efficiency given by Loimaranta.

\section{The Central Value}

We consider the equation

$$
E\left[X_{\tau}(\lambda)\right]=E\left[Y_{\tau}(\lambda)\right]
$$

which expresses the equality between the bonus-malus premium and the risk premium for a risk $\lambda$ in $[0, \tau[$. Because of the relations (4) equation (32) has at least one solution $\lambda_{\tau}^{*}$. We call a solution $\lambda_{\tau}^{*}$ of (32) a central value of the bonus-malus system in $[0, \tau[$.

We assume now that $w_{t}(\lambda)$ and $C_{t}(\lambda)$ are independent of $\lambda$ and we shall show that the central value in $\left[0, \tau\left[\right.\right.$ is unique if $e_{\tau}(\lambda)<I$ for all $\lambda$.

From (II) we have

$$
d \ln E\left[X_{\tau}(\lambda)\right]=e_{\tau}(\lambda) d \ln \lambda
$$


which gives if we integrate with $\lambda_{\tau}^{*}$ as initial value

$$
E\left[X_{\tau}(\lambda)\right]=E\left[X_{\tau}\left(\lambda_{\tau}^{*}\right)\right] e e^{\int_{\tau}^{\lambda} e_{\tau}(\lambda) d l n \lambda}
$$

where

$$
E\left[X_{\tau}\left(\lambda_{\tau}^{*}\right)\right]=E\left[Y_{\tau}\left(\lambda_{\tau}^{*}\right)\right]=\frac{\lambda_{\tau}^{*}}{\lambda} E\left[Y_{\tau}(\lambda)\right]
$$

so that

$$
E\left[X_{\tau}(\lambda)\right]= \begin{cases}E\left[Y_{\tau}(\lambda)\right] e^{\hat{j}^{*}}\left[1-e_{\tau}(\lambda)\right] d l n \lambda & \lambda \leq \lambda_{\tau}^{*} \\ E\left[Y_{\tau}(\lambda)\right] e^{-\lambda_{\tau}^{\lambda}\left[1-e_{\tau}(\lambda)\right] d l n \lambda} & \lambda \geq \lambda_{\tau}^{*}\end{cases}
$$

If $e_{\tau}(\lambda)<\mathrm{I}$ for all $\lambda$ we have thus that $\lambda_{\tau}^{x}$ is unique and that

$$
E\left[X_{\tau}(\lambda)\right] \gtreqless E\left[Y_{\tau}(\lambda)\right] \quad \text { if } \lambda \lesseqgtr \lambda_{\tau}^{*}
$$

Now we make some assumptions that will permit us to rewrite equation (32) in an easier form. As in section 3.3. we assume that $b_{t}(j)=\alpha^{t} b_{j}, w_{t}=(\mathrm{I}-\rho)^{t}$ and $\theta \leq \mathrm{I}$, where $\theta=\beta \alpha(\mathrm{I}-\rho)$. Further we assume that the evolution in time of the average claim cost can be given in the form $C_{t}(\lambda)=\gamma^{t} C$, with $C=C_{0}$ the average cost at constant price and $\gamma \geq \mathrm{I}$ the price index of claims. Hereby we put $\varepsilon=\beta \gamma(I-\rho)$ and take that $\varepsilon \leq I$. The central value in $\left[0, \tau\left[, \lambda_{\tau}^{*}\right.\right.$, is then the solution of the equation

$$
b(\lambda) \sum_{i=0}^{\tau-1} \theta^{t}+g_{s, \tau-1}(\lambda)=\lambda C \sum_{t=0}^{\tau-1} \varepsilon^{t}
$$

In particular we have that $\lambda_{1}^{*}=\frac{b_{s}}{C}$.

We call central value in $[0, \infty[$

$$
\lambda^{*}=\lim _{\tau \rightarrow \infty} \lambda_{\tau}^{*}
$$

and we distinguish the following cases. In the case $\theta<\mathrm{I}, \varepsilon<\mathrm{I}$ we have from (35) that $\lambda^{*}$ is the solution of the equation

$$
\frac{b(\lambda)}{\mathrm{I}-\theta}+g_{s}(\lambda)=\frac{\lambda C}{\mathrm{I}-\varepsilon}
$$

For $\theta=\mathrm{I}, \varepsilon<\mathrm{I}$ we have that $\lambda^{*} \rightarrow \infty$, while for $\theta<\mathrm{I}, \varepsilon=$ I holds $\lambda^{*} \rightarrow 0$. Finally in the case $\theta=\varepsilon=I$ we obtain that $\lambda^{*}$ is the solution of

$$
b(\lambda)=\lambda C
$$


The solution of this last equation corresponds with the concept central value as introduced by Loimaranta.

\section{APPENDIX}

We shall prove that the systems (29) and (3I) of $n+$ I equations in $n$ unknown have an unique solution.

5.I. We make use of the following two lemmas, in which o denotes the zero matrix and $I$ the unit matrix.

\section{Lemma I}

If $Q$ is a square matrix and $Q^{k}$ tends to $O$ as $k$ tends to infinity, then

$$
\operatorname{det}(I-Q) \neq 0
$$

and $(I-Q)^{-1}=I+Q+Q^{2}+\ldots=\sum_{k=0}^{\infty} Q^{k}$

\section{Proof}

see e.g. Kemeny and Snell p. 22.

\section{Lemma 2}

If to the $x$-th row (column) of the blocks of a partioned matrix $Q$ we add the $y$-th row (column) multiplied on the left (right) by a rectangular matrix $R$ of the corresponding dimensions, then the rank of $Q$ remains unchanged under this transformation and, if $Q$ is a square matrix, the determinant of $Q$ is also unchanged.

\section{Proof}

see e.g. Gantmacher p. 45.

We introduce the following matrix notations

$A: \mathrm{I} \times n$ matrix with elements $a_{i}(\lambda)$

$B: n \times$ I matrix with elements $b_{i}$

$G: n \times$ I matrix with elements $g_{i}(\lambda)$

$P: n \times n$ matrix with elements $p_{i j}(\lambda)$

$E: n \times$ I matrix with all elements equal to I

$D=b(\lambda) E: n \times$ I matrix with all elements equal to $b(\lambda)$

$M=E A: n \times n$ matrix whose rows are all identical and equal to $A$ 
According to (I5), (I6) and (I8) we have then

$$
\begin{aligned}
& \lim _{k \rightarrow \infty} P^{k}=M \\
& A P=A, A E=\mathrm{I} \\
& A B=b(\lambda)
\end{aligned}
$$

5.2. We now prove that the system (29) has an unique solution. In matrix notations this system becomes

$$
\left\{\begin{array}{l}
(\theta P-I) G=D-B \\
A G=0
\end{array}\right.
$$

The necessary and sufficient conditions for an unique solution are

$$
\operatorname{rank}\left[\begin{array}{c}
\theta P-I \\
A
\end{array}\right]=n, \operatorname{det}\left[\begin{array}{cc}
\theta P-I & D-B \\
A & 0
\end{array}\right]=0
$$

According to lemma 2 we have

$$
\operatorname{rank}\left[\begin{array}{c}
\theta P-I \\
A
\end{array}\right]=\operatorname{rank}\left[\begin{array}{c}
\theta(P-M)-I \\
A
\end{array}\right]
$$

where we have substracted from the first row the last row multiplied on the left by $\theta E$. Since for each power $q$ holds $M^{q}=M$ we have $(P-M)^{k}=P^{k}-M$. From $\lim P^{k}=M$ it follows then that $\lim [\theta(P-M)]^{k}=0$ for each $\vec{\theta}^{\infty} \leq \mathrm{I}$ and we have that $\operatorname{det}[\theta(P-M)-I] \neq 0$ according to lemma. $\mathrm{I}$. This shows that the coefficient-matrix has rank $n$.

To prove that the determinant in (40) is zero we make the following transformations:

$$
\begin{aligned}
& \operatorname{det}\left[\begin{array}{cc}
\theta P-I & D-B \\
A & 0
\end{array}\right]=\operatorname{det}\left[\begin{array}{cc}
\theta(P-M)-I & D-B \\
A & 0
\end{array}\right] \\
= & \operatorname{det}\left[\begin{array}{cc}
\theta(P-M)-I & D-B \\
0 & -A[\theta(P-M)-I]^{-1}(D-B)
\end{array}\right] \\
= & -A[\theta(P-M)-I]^{-1}(D-B) \cdot \operatorname{det}[\theta(P-M)-I]
\end{aligned}
$$

We have now

$$
\begin{aligned}
-A[\theta(P-M)-I]^{-1} & =A[I-\theta(P-M)]^{-1} \\
& =A \sum_{k=0}^{\infty}[\theta(P-M)]^{k}
\end{aligned}
$$




$$
\begin{aligned}
& =A\left[I+\sum_{k=1}^{\infty} \theta^{k}\left(P^{k}-M\right)\right] \\
& =A+\sum_{k=1}^{\infty} \theta^{k} A\left(P^{k}-M\right) \\
& =A
\end{aligned}
$$

with $A(D-B)=0$, which completes the proof.

5.3. For the system (3I) we have

$$
\left\{\begin{array}{l}
(\theta P-I) G^{\prime}=D^{\prime}-\theta P^{\prime} G \\
A G^{\prime}=-A^{\prime} G
\end{array}\right.
$$

where a quote indicates derivation with respect to $\lambda$.

The necessary and sufficient conditions for an unique solution are

$$
\operatorname{rank}\left[\begin{array}{c}
\theta P-I \\
A
\end{array}\right]=n, \operatorname{det}\left[\begin{array}{cc}
\theta P-I & D^{\prime}-\theta P^{\prime} G \\
A & -A^{\prime} G
\end{array}\right]=\mathrm{o}
$$

The first condition is the same as in section 5.2. and is thus satisfied. For the second condition we obtain after some transformations

$$
\begin{aligned}
& \operatorname{det}\left[\begin{array}{cc}
\theta P-I & D^{\prime}-\theta P^{\prime} G \\
A & -A^{\prime} G
\end{array}\right] \\
& =\left\{-A^{\prime} G-A[\theta(P-M)-I]^{-1}\left[D^{\prime}-\theta P^{\prime} G+\theta E A^{\prime} G\right]\right\} . \\
& \operatorname{det}[\theta(P-M)-I]
\end{aligned}
$$

The proof follows now from

$$
\begin{aligned}
-A^{\prime} G+A D^{\prime}-\theta A P^{\prime} G+\theta A^{\prime} G & =-A^{\prime} G+A D^{\prime}+\theta A^{\prime} P G \\
& =A^{\prime}[(\theta P-I) G+B] \\
& =A^{\prime} D=0 .
\end{aligned}
$$

\section{ReFERENCES}

Gantmacher, F. R., (I960), The Theory of Matrices, Vol. I, Cheisea Publishing Company, New York.

Kemeny, J. G. and SNeLL J. L., (I960), Finite Markov Chains, D. Van Nostrand Company, New York.

Lemaire, J., (1975), Si les assurés connaissaient la programmation dynamique... Bulletin de l'Association Royale des Actuaires Belges, $\mathrm{N}^{0} 7 \mathrm{o}, \mathrm{pp} \cdot 54-63$.

Lemaire, J., (I976), Driver versus Company-Optimal Behaviour of the Policy Holder, Scandinavian Actuarial Journal, No 1976-4, pp. 209-2 I9. Loimaranta, K., (I972), Some asymptotic properties of bonus systems, Astin Bulletin, Vol. VI, Part 3, pp. 233-245. 